\title{
Powder preparation and compaction behaviour of fine-grained Y-TZP
}

\author{
W. F. M. GROOT ZEVERT, A. J. A. WINNUBST*, G. S. A. M. THEUNISSEN, \\ A. J. BURGGRAAF \\ University of Twente, Faculty of Chemical Technology, Laboratory for Inorganic Chemistry, \\ Materials Science and Catalysis, P.O. Box 217, 7500 AE Enschede, The Netherlands
}

\begin{abstract}
Two wet chemical preparation methods are described for yttria-doped tetragonal zirconia powders. Both methods yield powders with an extremely small crystallite size $(8 \mathrm{~nm})$ and a narrow size distribution. The agglomerate and aggregate structure of these powders have been investigated by several techniques. Gel precipitation from an alkoxide solution in water ("alkoxide" synthesis) results in a ceramic powder with irregular-shaped weak and porous agglomerates, which are built up from dense aggregates with a size of $18 \mathrm{~nm}$. Gel precipitates formed from a metal-chloride solution in ammonia ("chloride" synthesis) do not contain aggregates. Both types of agglomerate are fractured during isostatic compaction. Hydrolysis and washing under (strong) basic conditions probably decrease the degree of aggregation. The aggregate morphology and structure are key parameters in the microstructure development during sintering of a ceramic. Several characteristics of these powders are compared with those of a commerical one (Toyo Soda TZ3Y).
\end{abstract}

\section{Introduction}

Ceramic materials which consist of $100 \%$ yttria-doped tetragonal zirconia polycrystals (Y-TZP) exhibit a high strength and toughness compared to other ceramics. For zirconia doped with $3 \mathrm{~mol} \% \mathrm{Y}_{2} \mathrm{O}_{3}$ the ceramic grain size must be less than $0.8 \mu \mathrm{m}$ to remain fully tetragonal [1]. However this critical grain size is much smaller (about $0.3 \mu \mathrm{m}$ ) when the ceramic has to remain tetragonal after ageing in water at elevated temperatures $[2,3]$. In order to obtain dense and pure tetragonal zirconia ceramics with these small grain sizes, special requirements must be fulfilled by the powders used for the sintering process. These ceramic powders must be homogeneous in composition and highly sinteractive (low sintering temperature) in order to decrease grain growth during sintering. They can be prepared by means of wet chemical methods such as sol-gel or gel precipitation. These fine grained (submicrometer) powders are not monodispersed but consist of microstructural elements due to clustering [4-8]. These elements are: (1) primary crystallites; (2) aggregates in which the primary crystallites are held together by neck areas which are formed by a reaction, e.g. sintering of the powder by surface diffusion or even diffusion and precipitation at necks in the suspension [4]; (3) agglomerates. The aggregates or the individual primary crystallites are held together in an agglomerate structure by relatively weak (e.g. Van der Waals, capillary) attractive forces.

During wet-chemical preparation these crystallite "clusters" ( 2 and 3$)$ are essentially formed in the early stages of the preparation cycle [9]. The state of these clusters determines, for example, surface area, flow behaviour, densification processes during compaction and the final grain size in the ceramic. The clusters in the powder can be detrimental to sintering when they are still present in the green compact prior to sintering. In those types of compacts no homogeneous pore size distribution is present and the pores within the compact, which can be much larger than the crystallites, only disappear during sintering at extremely high temperatures or do not disappear but even grow $[5,10]$. These porosity inhomogeneities are a source for inhomogeneous sintering, which can give coarse-grained regions in the sintered compact. Those inhomogeneities induce nonuniform sintering rates and back stress effects, which in turn create transient (or residual) stresses [11].

In studies of the microstructural development of cubic zirconia it has been found that the aggregates with a size of some tens of nanometres already sinter to full density at very low temperature (about $1170 \mathrm{~K}$ ) forming larger single crystallites [8]. These crystallites (size about $15 \mathrm{~nm}$ ) probably form the initial grain size with which the normal grain growth process starts and consequently the aggregate size is important for the final grain size of the dense ceramic. This relation is studied in the current programme to obtain dense ceramics with a grain size of $0.1 \mu \mathrm{m}$ and smaller.

In this paper a detailed study is made of the change in agglomerate morphology during compaction of fine-grained and weakly agglomerated powders. Three different ceramic powders were used. Two powders prepared by hydrous-gel-precipitation methods and a commercially available powder (Toyo Soda TZ3Y).

* Author to whom all correspondence should be addressed. 


\section{Experimental procedure}

\subsection{Powder preparation}

The zirconia-yttria powders were prepared by two different gel-predipitation techniques. Both implied the hydrolysis of a diluted $\mathrm{Zr}-\mathrm{Y}$ precursor solution in an excess of hydrolysing agent.

For one method (the "alkoxide" method), freshly prepared $\mathrm{Zr}$-t-amyloxide and $\mathrm{Y}$-isopropoxide were used. A $0.2 \mathrm{M}$ solution of these alkoxides in benzene was slowly added to a large excess of water (molar ratio $\mathrm{H}_{2} \mathrm{O}$ : metal $=250$ ). The hydrolysis was performed in a dispersion turbine reactor as described previously [8]. After hydrolysis, the gel was washed three times with water in order to remove organic solvents. This hydrous gel was filtered and wet-milled with ethanol in plastic containers using teflon balls. Subsequently the gel was washed three times with an excess of ethanol in order to remove the free water within the gel. All the washing steps were performed with large amounts of washing agent (gel concentration about $0.1 \mathrm{M}$ ) using a high-energy disc turbine [8].

The precursor for the second method consists of commercial $\mathrm{ZrCl}_{4}$ (Merck) and $\mathrm{YCl}_{3}$ (Cerac) which were dissolved in $\mathrm{HCl}(0.4 \mathrm{M})$. The precursor solution was added slowly to an excess of a $25 \%$ ammonia solution. This method is called the "chloride" method. During hydrolysis the $\mathrm{pH}$ remained at 11 or more. The hydrolysis and washing procedure were performed in the same reactor as described for the alkoxide method. Washing of the chloride-derived gel with water must be performed very thoroughly in order to remove chlorides from the solution. For that reason the gel was washed about eight times in a solution with a gradually decreasing amount of ammonia. Subsequently the gel was washed three times with an alcohol to remove free water in the same way as described for the "alkoxide" method.

Both gels ("alkoxide" and "chloride" derived) were filtered, dried in air for $16 \mathrm{~h}(390 \mathrm{~K})$, dry-milled (in a plastic container equipped with teflon balls) and calcined at $820 \mathrm{~K}$ for $2 \mathrm{~h}$ after which they were drymilled again.

The microstructure of the alkoxide and chloride powders were compared with a commercially available powder with about the same composition (Toyo Soda/ TOSOH; TZ3Y).

\subsection{Characterization}

Several techniques were used to characterize the powders. The amount of zirconia and yttria was analysed by X-ray fluorescence spectrometry using a Philips PW 1410 spectrometer. The amount of chloride was determined by dissolving the powder in sulphuric acid during long-term refluxing. In order to counteract the formation of gaseous hydrochloric acid, a cold finger was used during refluxing. The solution was afterwards titrated with an $\mathrm{AgNO}_{3}$ solution.

A Philips X-ray diffractometer PW 1710 using $\mathrm{Cu} K \alpha$ radiation was used to determine the phasecomposition and the primary crystallite size of the powder. The Scherrer equation was used to determine the crystallite size from X-ray line broadening measurements. In this case correction for instrumental broadening and $K \alpha$-splitting has been applied. The crystallite-size of the as-calcined powders were also verified by means of TEM (Jeol 200 CT).

The specific surface area was determined according to the BET method using the equipment as reported by Bosch and Peppelenbos [12].

The tap density was measured by vibrating a testtube containing the powder until the powder volume remained constant. The powders were isostatically pressed up to $500 \mathrm{MPa}$. Compaction behaviour was tested by measuring the density as a function of compaction pressure. The overall density was determined using the Archimedes technique (in mercury).

The pore-size distribution and the pore volume in the compacts were calculated from a nitrogen adsorption/desorption isotherm at $77 \mathrm{~K}$ using a Carlo Erba Sorptomatic Series 1800. The desorption branch was used according to the method described by Dollimore and Heal [13]. Pores with radii larger than about $10 \mathrm{~nm}$ were determined by mercury-intrusion porosimetry. These measurements were performed with a Carlo. Erba Porosimeter Series 200 at pressures up to $200 \mathrm{MPa}$.

The as-calcined powders were isostatically pressed to $400 \mathrm{MPa}$ before sintering. For these sintering experiments the temperature was raised at a rate of $1 \mathrm{~K} \mathrm{~min}^{-1}$ to the desired temperature. At this temperature the compact was sintered for $10 \mathrm{~h}$ in air.

\section{Results and discussion}

The X-ray fluorescence measurements showed that the composition of the powders was near to the nominal composition: $\mathrm{ZrO}_{2}+3 \mathrm{~mol} \% \mathrm{Y}_{2} \mathrm{O}_{3}$ (Table I). The phase analysis by $\mathrm{X}$-ray diffraction revealed that only traces of monoclinic zirconia were observed in the as-calcined "alkoxide" and "chloride" powders. In contrast the Toyo Soda powder clearly contained monoclinic and cubic crystallites.

The amount of chloride in the as-calcined powders is important because there is an indication that sintering starts at higher temperatures when the ceramic powder contains more chloride. For undoped zirconia made by the same "chloride" method it was shown that the temperature at which transformation from tetragonal to monoclinic zirconia takes place decreases with increasing amount of chloride in the as-calcined powder [14].

The specially developed gel-washing procedure for the "chloride" method showed good results in decreasing the amount of chloride in the resulting calcined powder. The chloride concentration in the chloride powder was four times larger when, after hydrolysing in ammonia, the gel was only washed with water instead of an ammonia/water solution.

The primary crystallite sizes of the as-calcined chloride and alkoxide powders were comparable $(8 \mathrm{~nm})$. A TEM picture of the chloride powder is shown in Fig. 1. The commercial Toyo Soda powder contained crystallites which were about four times as large $(34 \mathrm{~nm})$. The surface area of the Toyo Soda powder was much lower than the $S_{\mathrm{BET}}$ of the chloride and alkoxide powders. The reason for the observed 


\begin{tabular}{lllllr}
\hline Synthesis & $\begin{array}{l}\text { Composition } \\
\left(\mathrm{mol} \% \mathrm{YO}_{1.5}\right)\end{array}$ & $\begin{array}{l}\mathrm{Cl}^{-} \text {content } \\
(\mathrm{wt} \%)\end{array}$ & $\begin{array}{l}\text { Crystal structure } \\
(\%)\end{array}$ & $\begin{array}{l}\text { Crystallite size, } d \\
(\mathrm{~nm})\end{array}$ & $\begin{array}{l}S_{\text {BET }} \\
\left(\mathrm{m}^{2} \mathrm{~g}^{-1}\right)\end{array}$ \\
\hline alkoxide & 5.9 & $<0.001$ & $\mathrm{t}>99$ & 8 & 101 \\
chloride & 5.7 & 0.015 & $\mathrm{t}>99$ & 8 & 124 \\
Toyo Soda & 5.5 & 0.010 & $\mathrm{~m}=16$ & 34 & 21 \\
& & & $\mathrm{c}=10$ & \\
$\mathrm{t}=74$ & & \\
\hline
\end{tabular}

$\mathrm{t}=$ tetragonal, $\mathrm{m}=$ monoclinic, $\mathrm{c}=$ cubic.

differences in the $S_{\mathrm{BET}}$. values between the three powders will be discussed later.

The powders were isostatically pressed up to $500 \mathrm{MPa}$. During compaction overall density measurements were made on several compacts. These relative densities were plotted as a function of the logarithm of the applied pressure (Fig. 2). The curves show two linear parts (parts 1 and 2) with a point of intersection at pressure, $P_{y}$. The linear part at high pressures (part 2, Fig. 2) can be described by the empirical equation

$$
\rho-\rho_{\mathrm{Y}}=m \ln \left(P / P_{\mathrm{y}}\right)
$$

where $m$ is a constant, $\varrho$ the density and $P$ the pressure. The subscript y stands for the yield point. The values of $P_{y}$ and $m$ are given in Table III. $M$ is probably a constant which is related to the microstructure of the powder. The calculated values for $m$ (Table III) show large differences. Halloran $[5,6]$ used the same equation but did not prove the applicability nor report any $m$ values.

According to Van de Graaf et al. [8] the intersection $P_{\mathrm{y}}$ is the strength of the largest microstructural element. After compaction at a pressure around $P_{\mathrm{y}}$ these elements are all gradually fragmentated, while they are only rearranged at pressures below $P_{y}$. These elements are called the agglomerates. However, if a powder is granulated the granules are the first elements to be broken. The Toyo Soda powder is a granulated powder as is confirmed by TEM (Fig. 3). Clearly this powder consists of spherical-shaped granules. The $P_{\mathrm{y}}$ values differ significantly for the powders studied (Table III). For the chloride powder, the $P_{\mathrm{y}}$ value (intersection) is much larger than for the other two. In this paper the density, packing capability (morphology) and strength of the agglomerates will be discussed.

In order to obtain information about the density of the agglomerates the pore-size distributions after isostatic compaction of the powders has to be considered (see Fig. 4). The range of the pore radii within the powder compacts can be divided into three sections as indicated in Fig. 4: (1) $R \leqslant 3 \mathrm{~nm}$, the intra-aggregate pores (pores between the crystallites); (2) $3 \leqslant \mathrm{R}<100 \mathrm{~nm}$, the inter-aggregate pores; (3) $R>100 \mathrm{~nm}$, the inter-agglomerate pores or the intergranular pores in case of the Toyo Soda powder. The

TABLE II Packing properties of the as-calcined powders

\begin{tabular}{llcl}
\hline Synthesis & TD $(\%)$ & SD $(\%)$ & $\Delta(\mathrm{TD}-\mathrm{SD})(\%)$ \\
\hline alkoxide & 12 & 5 & 7 \\
chloride & 19 & 17 & 2 \\
Toyo Soda & 22 & 22 & 0 \\
\hline
\end{tabular}

agglomerate or granular density was calculated from the sum of the pore volumes of the inter-and intraaggregate pores.

After compaction at $P=4 \mathrm{MPa}$ the agglomerates themselves remain intact, because for each powder the applied pressure is less than $P_{y}$ (Table III). The agglomerate density in these powder compacts is therefore the same as in the unpressed powder. The pores with a radius smaller than $R=100 \mathrm{~nm}$ (sections 2 and 3 in Fig. 4a) have to be taken into account for calculating the agglomerate density by means of the equation

$$
\varrho_{\text {agg. }}=[x /(x+y)] \times 100 \%
$$

where $x=1 / \varrho_{1}\left(\varrho_{t}\right.$ is the theoretical density) and $y$ is the volume (in units of gram powder) of the nitrogen which is adsorbed in the intra-agglomerate pores as determined by nitrogen adsorption/desorption measurements. The agglomerate densities obtained in this way are given in Table III. From these results it can be seen that the agglomerates of the alkoxide powder are more porous than the chloride agglomerates. The Toyo Soda granules are rather dense.

The difference in tap density values (TD) as given in Table II can be explained by the difference in agglomerate densities. A ceramic powder with a high agglomerate density shows a high tap density.

In order to obtain large compacts it is not only important to have dense agglomerates but also to have agglomerates with a good packing capability. The density of a powder after it is poured into a tube or mould without applying any external force is a good characteristic for the resulting packing capability of the powder. This density is determined by an extrapolation of the linear part of the density-compaction

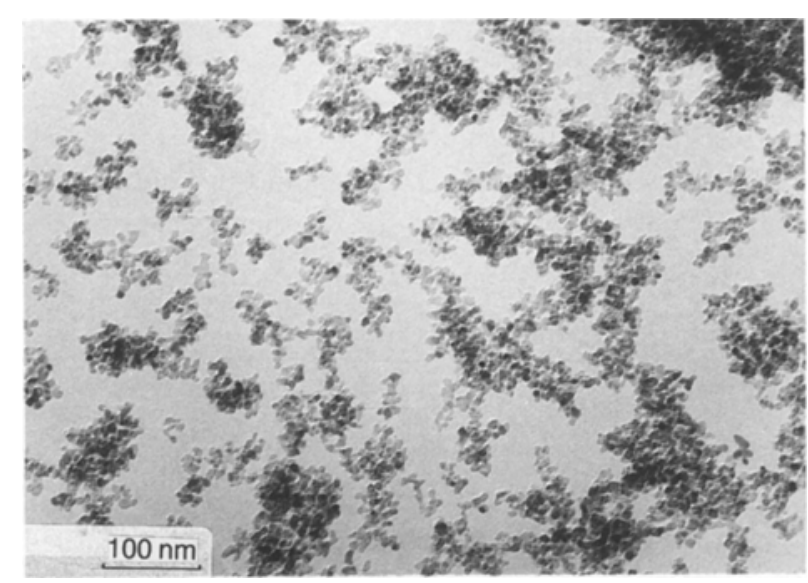

Figure I Transmission electron micrograph of a calcined "chloride" powder. 


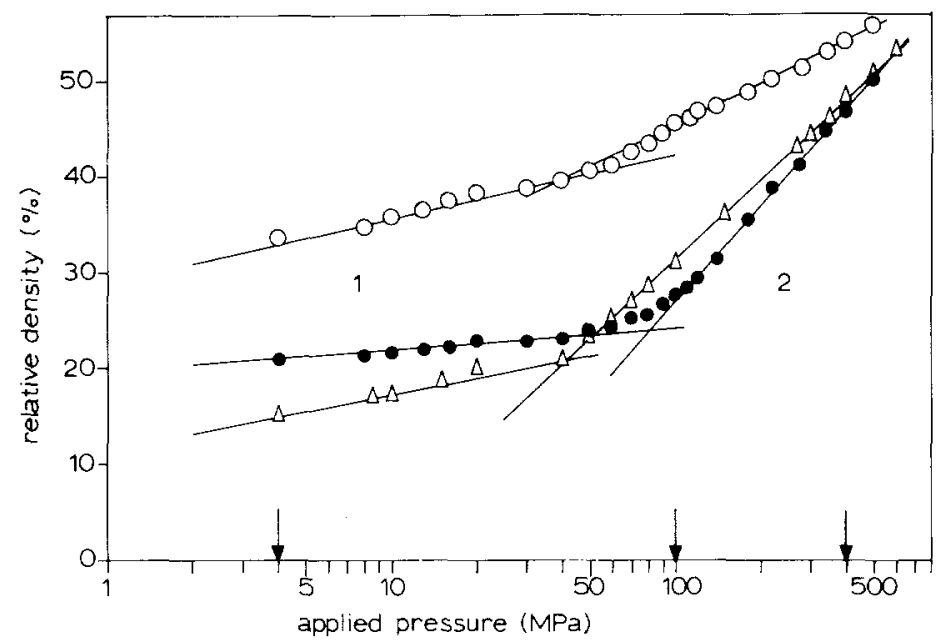

Figure 2 Densification behaviour of the $\mathrm{ZrO}_{2}-\mathrm{Y}_{2} \mathrm{O}_{3}$ powders during isostatic compaction. $\downarrow$ : Pore size distribution measured at these pressures. $(\Delta)$ Alkoxide, (O) Toyo Soda, (•) Chloride.

pressure curve at low pressures (part 1 in Fig. 2) down to $P=0.1 \mathrm{MPa}$. The density of this point (further referred to as SD) can deviate from the density obtained after putting some energy to the powder system by vibrating the tube. This latter density is called the tap density (TD). Values for both densities are given in Table II. From these results it is clear that SD and TD are dependent on the preparation method of the powder. Also a significant difference in SD and TD for the alkoxide powder is observed. These phenomena will be discussed now. A measure for good packing capability is the packing factor of the powder $(p)$ which is defined by Equation 3 according to Halloran $[5,6]$

$$
p=S D / \varrho_{\text {aggl. }}
$$

Here SD is used instead of TD because SD is a value of powder packing without any external force added during stacking. The $\mathrm{fcc}$ packing is the ideal situation, which leads to a $p$ value of 0.74 when spherical particles with uniform diameter are assumed. According to Fedors [15], however, a value of 0.63 is the most probable $p$ value when mechanical vibration is employed to a powder which consists of nondeformable particles with a uniform size. This $p$ value was determined by assuming that about one-half of the particles was packed in a (hexagonal) close packing $(=\mathrm{fcc})$ and the other half in a simple cubic array ( $=\mathrm{sc}$ with $p=0.52$ ). In Table III the $p$ values of the investigated powders are given. The $p$ values of the chloride and Toyo Soda powders are relatively high. This indicates that the agglomerates of the chloride powder and the granules of the Toyo Soda powder stack rather well. Probably the particles can move easily with respect to each other and are spherically shaped. This also explains why, for these two powders, the SD and TD values were about equal. The $p$ value for the alkoxide powder is rather low. The alkoxide agglomerates pack poorly prior to compaction. The difference in SD and TD for this powder was also rather large. Both effects indicate that the agglomerates of the alkoxide powder are irregularly shaped.

Another important characteristic of a powder agglomerate is its strength. Indicative for the agglomerate strength is the $P_{\mathrm{y}}$ value as given in Table III. The larger $P_{\mathrm{y}}$ value in the chloride powder indicates that the agglomerates in this powder are stronger than the agglomerates in the alkoxide powder or the Toyo Soda granules. For the chloride powder, however, the density-compaction curve is not linear between $P=50$ and $100 \mathrm{MPa}$ (see Fig. 2). These two values probably represent the lowest and highest strength of the agglomerates. The overall density at the point of maximal agglomerate strength $(P=100 \mathrm{MPa})$ is in good agreement with the agglomerate density as given in Table III. For the alkoxide and Toyo Soda powder the density-compaction curve is clearly divided into two sharply separated linear parts. This indicates that the maximum strength for the agglomerates/granules of the powder is very close to its average strength. So the agglomerate strength in these two powders is rather uniform. The relative densities of the powder compacts at the pressure at which all agglomerates are fragmented (yielding point in Fig. 2) is in good agreement with the agglomerate densities (Table III). This is an indication, that the internal agglomerate (or granule) structure remains the same at pressures below the yielding point.

In order to investigate the change in microstructure during compaction, the pore-size distributions are determined from powder compacts pressed at 100 and $400 \mathrm{MPa}$ (Figs $4 \mathrm{~b}$ and c). After compaction at

TABLE III Agglomerate properties

\begin{tabular}{llllll}
\hline Synthesis & Agglomerate strength, & $\begin{array}{l}\text { Agglomerate density } \\
\rho \text { aggl (\%) }\end{array}$ & $m$-value* $(\%)$ & SD-value (\%) & $p$ factori \\
& $P_{\text {y }}(\mathrm{MPa})$ & 22 & 11 & 5 & 0.22 \\
alkoxide & 45 & 30 & 14 & 17 & 0.56 \\
chloride & 80 & $36 \neq$ & 7 & 22 & 0.61 \\
Toyo Soda & 40 & & & & \\
\hline
\end{tabular}

IGranule density for the Toyo Soda powder.

*According to Equation 1.

$\uparrow$ According to Equation 3. 


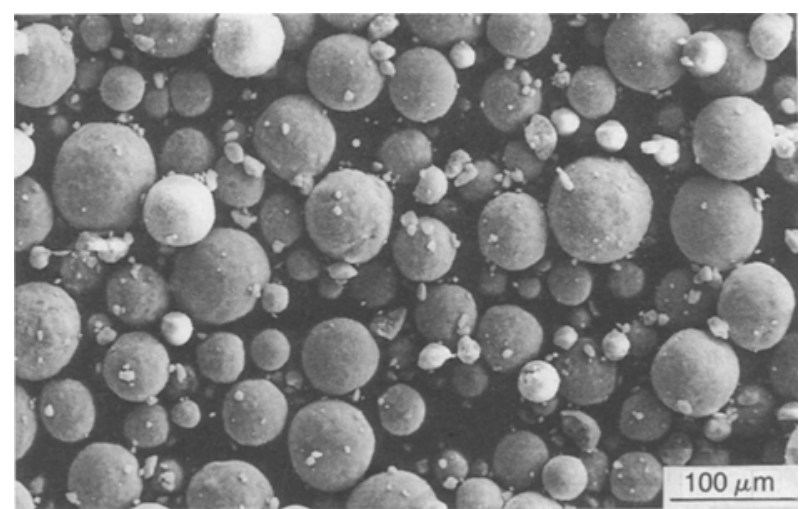

Figure 3 Scanning electron micrograph of a Toyo Soda powder.

$100 \mathrm{MPa}$ the large pores which are ascribed to the inter-agglomerate pores (section 3, Fig. 4) disappear. This again is evidence that the strength of all agglomerates or granules is less than (or equal to) $100 \mathrm{MPa}$.

In Table IV the mean pore sizes of the inter- and intra-aggregate pores are given as functions of the applied pressure. For the Toyo Soda Powders, Lecloux et al. [7] reported an inter-aggregate pore size of $28 \mathrm{~nm}$, which is in agreement with the inter-aggregate pore size after compaction at $4 \mathrm{MPa}$ (see Fig. $4 \mathrm{a}$ and Table IV). After isostatic compaction at $100 \mathrm{MPa}$ the alkoxide and Toyo Soda powder compacts show smaller pores in section 2, Fig. 4 (the inter-aggregate pores) if compared with compaction at $4 \mathrm{MPa}$. This decrease in inter-aggregate pore size takes place between $P_{\mathrm{y}}$ and $100 \mathrm{MPa}$. This effect is not observed in the chloride powder because the difference between $P_{\mathrm{y}}$ and $100 \mathrm{MPa}$ is small and because the maximum strength of the chloride agglomerates is $100 \mathrm{MPa}$. So after compaction at $100 \mathrm{MPa}$ all the agglomerates of the chloride powder are rearranged but the internal agglomerate structure remains the same.

A decrease in inter-aggregate pore size is observed in all types of powder by further compaction up to $P=400 \mathrm{MPa}$ (see Table IV and Fig. 4c). The pores in the alkoxide and chloride powders are as small as $R$ $=3$ to $4 \mathrm{~nm}$ and in the Toyo Soda compact $R=$ $17 \mathrm{~nm}$. The Toyo Soda compacts did not clearly show any pores in region 1 . The pores in this region can probably be ascribed to pores within the aggregates, the intra-aggregate pores. This will now be discussed.

The presence of aggregates can be demonstrated in the following way. As shown previously, the primary crystallites of the alkoxide and chloride powder were comparative in size $(8 \mathrm{~nm})$. The Toyo Soda powder had a crystallite size of $34 \mathrm{~nm}$ (see Table I). These crystallite sizes were determined by X-ray line broadening measurements. Crystallite sizes can also

TABLE IV Pore-size distribution data for various pressures and sections 1 and 2

\begin{tabular}{|c|c|c|c|c|c|c|}
\hline \multirow[t]{2}{*}{ Synthesis } & \multicolumn{2}{|l|}{$4 \mathrm{MPa}$} & \multicolumn{2}{|c|}{$100 \mathrm{MPa}$} & \multicolumn{2}{|c|}{$400 \mathrm{MPa}$} \\
\hline & $1(\mathrm{~nm})$ & $2(\mathrm{~nm})$ & $1(\mathrm{~nm})$ & $2(\mathrm{~nm})$ & $1(\mathrm{~nm})$ & $2(\mathrm{~nm})$ \\
\hline Alkoxide & $0-3$ & 15 & $0-3$ & 10 & $0-3$ & 4 \\
\hline Chloride & $*$ & 7 & $0-3$ & 7 & $0-3$ & 3 \\
\hline Toyo Soda & $*$ & 28 & $*$ & 24 & $*$ & 17 \\
\hline
\end{tabular}

* No pores detected in this section.

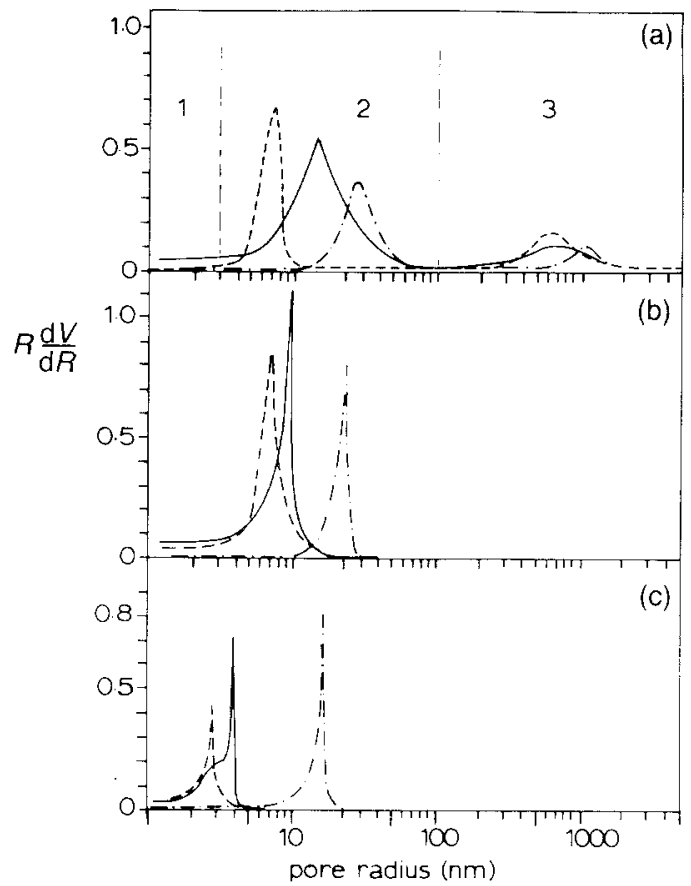

Figure 4 Pore-size distribution within the powder compacts after isostatic pressing at: (a) $4 \mathrm{MPa}$, (b) $100 \mathrm{MPa}$, (c) $400 \mathrm{MPa}$. (- ) Alkoxide, (- -) Chloride, (-.-..--) Toyo Soda.

be calculated from the specific surface area $\left(S_{\mathrm{BET}}\right)$. In that case an assumption is made that the total surface of a spherically shaped crystallite is accessible for the absorbed gas. The crystallite diameter $\left(d_{\mathrm{s}}\right)$ calculated from $S_{\mathrm{BET}}$ is

$$
d_{\mathrm{s}}=6 /\left(\varrho_{\mathrm{t}} S_{\mathrm{BET}}\right)
$$

where $Q_{1}$ is the theoretical density and $S_{\mathrm{BET}}$ the specific surface area. The crystallite sizes as determined by $\mathrm{X}$-ray line broadening $\left(d_{\mathrm{x}}\right)$ and the crystallite sizes as calculated from Equation $4\left(d_{s}\right)$ are given in Table $V$. The $d_{\mathrm{s}}$ value of the chloride powders fits well with the $d_{x}$ value, while for the alkoxide and Toyo Soda powders these values do not match. The difference in crystallite size value found with $\mathrm{X}$-ray line broadening and with $S_{\mathrm{BET}}$ can be explained by the fact that in these types of powder the primary crystallites are clustered and form an aggregate. In this way Argon, used for the BET measurement, cannot completely cover the crystallite surface. So these differences between $d_{\mathrm{s}}$ and $d_{x}$ give some indication of the degree of aggregation.

The size and structure of aggregates is also determined by the number of necks, which are formed by reaction (sintering) during calcination. According to Ramsay [16] the number of necks (and therefore an indication of the number of crystallites per aggregate)

TABLE V Aggregate properties

\begin{tabular}{|c|c|c|c|c|c|}
\hline \multirow[t]{2}{*}{ Synthesis } & \multirow{2}{*}{$\begin{array}{l}d_{x}(\text { XRLB }) \\
(\mathrm{nm})\end{array}$} & \multirow{2}{*}{$\begin{array}{l}d_{\mathrm{s}}(\mathrm{BET}) \\
(\mathrm{nm})\end{array}$} & \multirow{2}{*}{$\begin{array}{l}\text { Necks*, } \\
n\end{array}$} & \multicolumn{2}{|c|}{ Particles } \\
\hline & & & & $\mathrm{cp}$ & sc \\
\hline Alkoxide & 8 & 10 & 15 & 8 & 10 \\
\hline Chloride & 8 & 8 & 0 & - & - \\
\hline Toyo Soda & 34 & 47 & 96 & 42 & 50 \\
\hline
\end{tabular}

$*$ Necks $=$ number of necks within an aggregate.

$\uparrow$ particles $=$ number of particles per aggregate. $\mathrm{cp}=$ close packing assumed, $\mathrm{sc}=$ simple cubic packing assumed. 


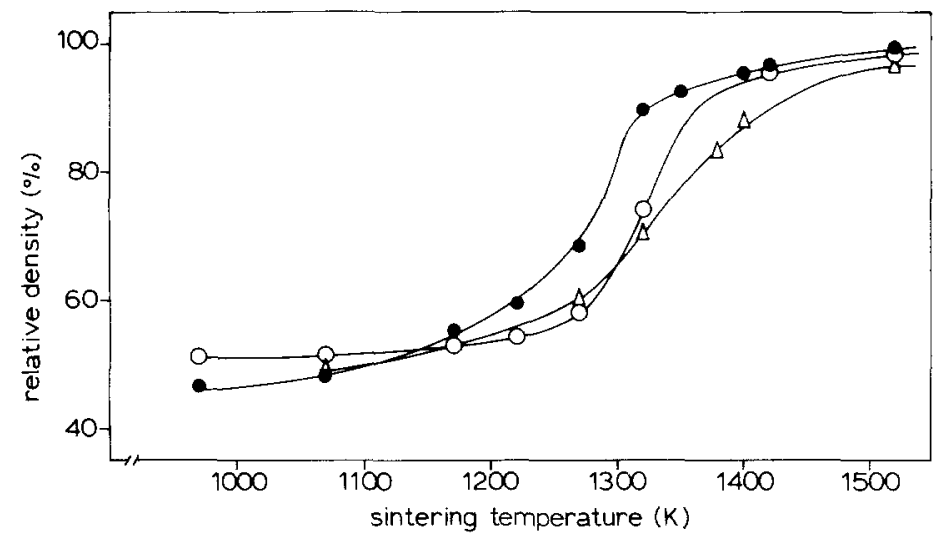

Figure 5 Densification behaviour of green compacts after stepwise sintering for $10 \mathrm{~h}$. (•) Chloride, $(\Delta)$ Alkoxide, (0) Toyo Soda.

could be calculated from the following equation

$$
n=2 d_{\mathrm{x}} / d_{\mathrm{Ar}}\left(1-S / S_{\mathrm{t}}\right)
$$

where $n$ is the number of necks, $d_{\mathrm{x}}$ the crystallite diameter obtained from X-ray line broadening measurements and $d_{\mathrm{Ar}}$ the diameter of an argon molecule. $S$ is the surface area attainable for argon (= the measured $S_{\mathrm{BET}}$ ). $S_{\mathrm{t}}$ is the theoretical surface area when it is assumed that each individual (spherical) crystallite can be completely covered with argon (according to Equation 4). There is a significant difference in the number of necks between the three powders (Table $V$ ). An estimation for the number of crystallites forming an aggregate could be made from the value of $n$. However, this number of crystallites depends on the packing-structure. In Table $\mathrm{V}$ the results are shown for two different types of packing: the close packing and the simple cubic packing. The close packing is the ideal packing for spherical particles, the simple cubic packing is far from ideal. The number of necks calculated for the chloride powder is zero (Table V), while in the alkoxide and Toyo Soda powders clearly necks are formed. These results, in combination with the fact that $d_{\mathrm{x}}$ fits with $d_{\mathrm{s}}$ (Table V), indicate that the chloride powder is aggregate-free. So for the chloride powder the pores found in section 1 and 2 of Fig. 4 are not intra-aggregate pores but inter-crystallite pores.

The Toyo Soda and alkoxide powders do contain aggregates. The Toyo Soda aggregates are built up from about 42 to 50 primary crystallites per aggregate and the alkoxide powder from about 9 to 10 primary crystallites per aggregate. Although the Toyo Soda powder contains aggregates (see Table $\mathrm{V}$ ) the compacts showed no pores which can be ascribed to intraaggregate pores (section 1, Fig. 4). This indicates that the packing of crystallites in the aggregate is very dense.

The pore-size distributions of the alkoxide compacts did show pores in Section 1 (see, for example, the shoulder in Fig. 4c). These pores are ascribed to intra-aggregate pores. The aggregate density, calculated from the pore volume within the aggregates, is about $70 \%$. Because an alkoxide aggregate consists of only 9 to 10 primary crystallites, the notion "aggregate density" is not very meaningful in this case.

The aggregate size can be calculated from the aggregate density and the number of crystallites within the aggregates. When spherically shaped aggregates are assumed the diameter of dense Toyo Soda aggre- gates is about $60 \mathrm{~nm}$ while the aggregate diameter in the alkoxide powder is about $18 \mathrm{~nm}$.

The reason why alkoxide powders contain aggregates and the chloride powders do not may originate in the difference in the wet-chemical preparation method. The methods of synthesis only differ in the type of precursors and hydrolysing agents used. In the alkoxide synthesis organic precursors are coprecipitated in water under neutral or slightly acidic conditions. In this $\mathrm{pH}$ range part of the precipitated yttria in the gel may dissolve and precipitate again at the point of contact between gel particles [17]. This may increase the number of necks between the gel particles. After calcination of the dried gel these necks form a rather strong aggregate. The hydrolysing conditions during a chloride synthesis are strongly basic $(\mathrm{pH}>11)$. Under these conditions yttria will not dissolve again after it is precipitated. In this way no aggregation will occur due to the effect mentioned above.

The sinterability of the green compacts (pressed at $400 \mathrm{MPa}$ ) were determined by stepwise sintering until a relative density of $95 \%$ or more was achieved. The sintercurve (Fig. 5) revealed that the chloride powder was the most sinteractive one: $95 \%$ dense after $10 \mathrm{~h}$ sintering at $1390 \mathrm{~K}$. The Toyo Soda and alkoxide powders were $95 \%$ dense after sintering for $10 \mathrm{~h}$ at 1415 and $1475 \mathrm{~K}$, respectively.

The shape of the sintercurve of the Toyo Soda and chloride compact is about the same: a region of about $140 \mathrm{~K}$ in which the compacts densify from 55 to $90 \%$. This probably means that the densification in these two powder compacts proceed along the same mechanism. The difference in temperature at which densification occurs between the chloride and Toyo Soda compacts may be ascribed to the difference in crystallite sizes of the starting powders $(8$ and $34 \mathrm{~nm}$, respectively).

The shape of the sintercurve of the alkoxide compact is somewhat different: densification starts at the same temperature as in the chloride compact $(1130 \mathrm{~K})$, but densification from 55 to $90 \%$ occurs in a temperature region of about $220 \mathrm{~K}$ which is more than in the case of the Toyo Soda and chloride compacts $(140 \mathrm{~K})$. The difference in sinteractivity between the chloride and alkoxide powder is mainly due to the presence of porous aggregates within the alkoxide powder. According to Van de Graaf [8] intra-aggregate pores in cubic zirconia become intra-aggregate pores during 
sintering at low temperatures $(<1170 \mathrm{~K})$. So the intra-aggregate sinteractivity is very high. In this temperature region the aggregates densify to "single crystallites". In the alkoxide powder the sinteractivity above $1170 \mathrm{~K}$ is therefore determined by these larger particles.

The sinter experiments have proved that the alkoxide powders need a higher temperature to achieve full densification than the chloride powders. This can lead to a smaller grain growth in the chloride compact and therefore to smaller grains in the dense ceramic. This is important in the synthesis of tetragonal zirconia ceramics with enchanced strength or increased resistance against a spontaneous phase transformation in a humid atmosphere at elevated temperature.

Investigations of the microstructural development, sintering behaviour and grain growth of these powder compacts will be discussed in a forthcoming paper [18].

\section{Conclusions}

1. Ultra-fine grained $\mathrm{Y}_{2} \mathrm{O}_{3}$ doped $\mathrm{ZrO}_{2}$ ceramic powders are prepared by two different hydrous gel precipitation techniques: the hydrolysis of metal alkoxides and metal chlorides. Both powders contain primary crystallites with a diameter of $8 \mathrm{~nm}$. This is about four times smaller than the primary crystallites of the commercial Toyo Soda powder $(34 \mathrm{~nm})$. Several powder properties are dependent on the wet-chemical preparation method.

2. The ceramic powders prepared by the hydrolysis of metal alkoxides consist of relatively weak, irregular shaped, porous agglomerates. These agglomerates are built up from porous aggregates which contain 9 to 10 crystallites and have a diameter of $18 \mathrm{~nm}$. The bimodal pore-size distribution within an alkoxide compact after isostatic pressing at $400 \mathrm{MPa}$ is explained by the presence of these porous aggregates.

3 . The ceramic powders prepared by the hydrolysis of a metal chloride solution consist of stronger, more regular shaped, and denser agglomerates. In these powders no aggregate structure is observed. This is confirmed by the presence of only one type of pore after compaction at $400 \mathrm{MPa}$ and by the similarity between the crystallite size and the equivalent BET crystallite diameter.

4. The commercial Toyo Soda powder consists of granules with a size of 30 to $50 \mu \mathrm{m}$ which are relatively weak, very regular shaped and rather dense. The granules are built up from dense aggregates. The powder contains no agglomerates other than these granules. This is confirmed by the presence of only two types of pore after compaction at $4 \mathrm{MPa}$. One is ascribed to inter-granular pores, the other to interaggregate pores. The dense aggregates have a diameter of $60 \mathrm{~nm}$.

5. The agglomerates in the chloride and alkoxide powder and the granules in the Toyo Soda powder are weak enough to collapse completely during compaction prior to sintering.

6. The microstructure of the chloride powder is a better start for densification during sintering compared to the alkoxide or Toyo Soda powder.

7. Hydrolysis and washing under strong basic conditions results in a powder which contains a lower degree of aggregation than hydrolysis in neutral or acidic conditions.

\section{Acknowledgements}

We thank Mrs A. Bongers for performing the nitrogen adsorption/desorption measurements. These investigations were partly supported by the. Innovative Research Programme on Technical Ceramics (IOPTK) with financial aid from the Dutch Ministry of Economic Affairs.

\section{References}

1. F. F. LANGE, J. Mater. Sci. 17 (1982) 240.

2. T. SATO and S. SHIMAdA, J. Amer. Ceram. Soc. 68 (1985) 356 .

3. A. J. A. WINNUBST, A. J. BURGGRAAF, "Advances in Ceramics, Vol. 24, Edited by S. Sorniya, N. Yamamoto and H. Yanagida (American Ceramic Society, Columbus, Ohio, 1989). p. 39.

4. D. E. NIESZ, R. B. BENNETT and M. J. SNYDER, Amer. Ceram. Bull. 51 (1972) 677.

5. J. W. HALlorAN, in "Advances in Ceramics", Vol. 9, edited by J.A. Mangels and G. L. Messing (American Ceramic Society, Columbus, Ohio, 1984) pp. 67-75.

6. J. W. HALLORAN, in "Ultrastructure Processing of Ceramics, Glasses and Composites", edited by L. L. Hench and D. R. Ulrich (Wiley New York, 1984) p. 404.

7. A. J. LECLOUX, P. VERLEYE, J. BRONCKART, F. NOVILLE, P. MARCHOT and J. P. PIRARD, Reactiv. Solids 4 (1988) 309.

8. M. A. C. G. VAN DE GRAAF, J. H. H. TER MAAT and A. J. BURGGAAF, J. Mater. Sci. 20 (1985) 1407.

9. M. A. C. G. VAN DE GRAAF and A. J. BURGGRAAF in "Advances in Ceramics", Vol. 12, edited by N. Claussen, M. Ruhle and A. H. Heuer, (American Ceramic Society, Columbus, Ohio, 1984) pp. 744-55.

10. M. A. C. G. VAN DE GRAAF, T. VAN DIRJK, M. A. DE JONGH, A. J. BURGGRAAF, in "Science of Ceramics", Vol. 9, edited by K. J. De Vries (Dutch Ceramic Society, The Netherlands, 1977) pp. 75-83.

11. A. G. EVANS, J. Amer. Ceram. Soc. 65 (1982) 497.

12. H. BOSCH and A. PEPpelenbos, J. Phys. E. Sci. Instrum. 10 (1977) 605.

13. D. DOLlimore and G. R. HEAL, J. Appl. Chem. 14 (1964) 109.

14. P. D. L. MERCERA, J. G. VAN OMMEN, E. B. M DOESBURG, A. J. BURGGRAAF and J. R. H. ROSS, Appl. Catal. 57 (1990) 127.

15. R. F. FEDORS, Powder Technol. 22 (1979) 71.

16. J. D. F. RAMSAY, ibid. 4 (1972) 29.

17. J. C. FARINAS, R. MORENO, J. REQUENA and J. S. MOYA, Materials Science and Engineering A109 (1989) 97.

18. G. S. A. M. THEUNiSSEN, A. J. A. WINNUBST, W. F. M. GROOT ZEVERT and A. J. BURGGRAAF, J. Amer. Ceram. Soc. submitted.

Received 8 November 1988

and accepted 10 April 1989 